\title{
On Mass-Thickness Contrast in Annular Dark-Field STEM-in-SEM Images
}

\author{
Jason Holm ${ }^{1}$ and Ryan White ${ }^{1}$ \\ 1. Applied Chemical and Materials Division, National Institute of Standards and Technology, Boulder, \\ United States.
}

A benefit to transmission imaging with low energy (i.e. $<30 \mathrm{keV}$ ) primary electrons compared to higher energy (i.e. $>100 \mathrm{keV}$ ) electrons is that some interactions between the probe electrons and the sample are more likely with decreasing energy. One advantage of the enhanced interaction is that the angular scattering distribution of electrons forward scattered through a sample is likely to broaden with decreasing primary electron energy. By lowering the primary electron energy, samples exhibiting massthickness variations may exhibit stronger contrast in some instances, particularly for very thin samples, or for samples comprising low atomic number elements that do not scatter electrons strongly. This is not always the case, however, and depending on the detector acceptance angle employed and the sample composition, images recorded using Scanning Transmission Electron Microscopy in a Scanning Electron Microscope (STEM-in-SEM) techniques can exhibit unanticipated and sometimes confusing contrast [1]. In fact, as will be shown, apparent contrast reversal can be observed even for ultrathin low atomic number samples.

In a recent article, a method to enable comprehensive acceptance angle control for STEM-in-SEM imaging was described [2]. Although comprehensive imaging control is key to extracting the most information from a sample, too much control can also elicit confusing images if the user is unaware of the imaging conditions and electron scattering behavior of their sample. In this contribution, we show that mass-thickness contrast in annular dark-field STEM-in-SEM images can change unexpectedly if the detector acceptance angle is very small and spans a narrow range. The apparent contrast reversal is a function of the detector acceptance angle, and recommendations for setting up imaging conditions for quantitative mass-thickness analyses are offered.

To those ends, an SEM equipped with focused ion beam (FIB) milling capability was used to deposit ultrathin pads of carbon and platinum on ultrathin carbon support films as shown in Figure 1 . A $5 \mathrm{keV}$ electron beam was used to deposit discrete pads of increasing thicknesses, and the FIB $\left(\mathrm{Ga}^{+}\right)$ functionality was used to mill a small hole adjacent to the pads so that background vacuum image intensity levels could be quantified. Several series of annular dark-field STEM images were recorded of the samples using two small apertures that enabled thin annular detector configurations. The platinum pads were imaged with an annular aperture having inner radius $R_{\mathrm{i}}=0.51 \mathrm{~mm}$ and outer radius $R_{\mathrm{o}}=0.78$ $\mathrm{mm}$, the carbon pads were imaged using an annular aperture with $R_{\mathrm{i}}=50 \mu \mathrm{m}$ and $R_{\mathrm{O}}=65 \mu \mathrm{m}$. Darkfield images were recorded over a broad range of acceptance angles, and angular distributions of the image intensities were quantified as shown in Figure 2a. In this way, both the acceptance angles at which the maximum image intensities are exhibited and the scattering angle regimes where the apparent contrast reversal occurs are easily discerned. Figure $2 \mathrm{~b}$ shows a summary of the most probable scattering angles indicated in Figure 2a. A comparison with thickness measurements made using electron energy loss techniques (not shown) concluded that image contrast at the most probable scattering angle is linearly proportional to the sample mass-thickness.

Figure 1 demonstrates that even the simplest of samples can produce images that can be challenging to 
interpret. At an acceptance angle of $363 \mathrm{mrad}$ (Fig. 1a), the contrast appears as might be anticipated based on conventional mass-thickness arguments: the thickest pad exhibits the strongest contrast, the thinnest pad exhibits the weakest. At shallower acceptance angles (Fig. 2c) the contrast can be opposite to what might be anticipated: the thinnest pad exhibits the strongest contrast and the thickest exhibits the weakest. At intermediate acceptance angles (Fig. 2b), neither the thickest nor thinnest pads exhibit the strongest contrast. Similar behavior is exhibited by the ultrathin carbon films. Most of the contrast change occurs because of the broadened angular distribution of the forward scattered electrons and the use of small annular apertures that enable only a narrow fraction of the forward scattered electrons to form the images.

The results shown in Figure 2 suggest that when using a narrow annular aperture, the most probable scattering angles (represented by the peaks of the distribution curves) vary directly with pad thickness. Quantitative mass-thickness analyses should be performed at these points under these imaging conditions. Qualitative analyses should be performed at acceptance angles greater than approximately $300 \mathrm{mrad}$.

\section{References:}

[1] V. Morandi and P. Merli, J. Appl. Phys. 101 (2007) 114917.

[2] J. Holm and R. Keller, Ultramicroscopy 167 (2016) 43-56.

[3] This work is a contribution of the US Government and is not subject to United States copyright.

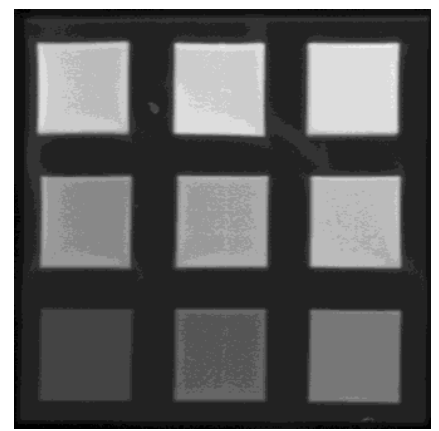

(a)

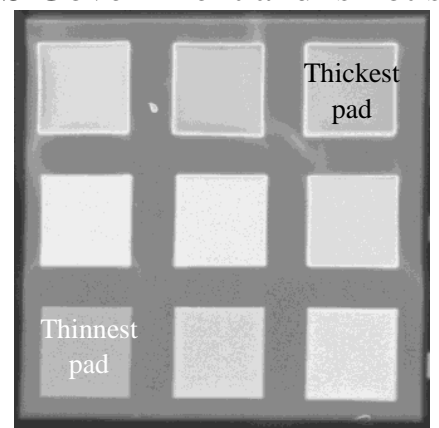

(b)

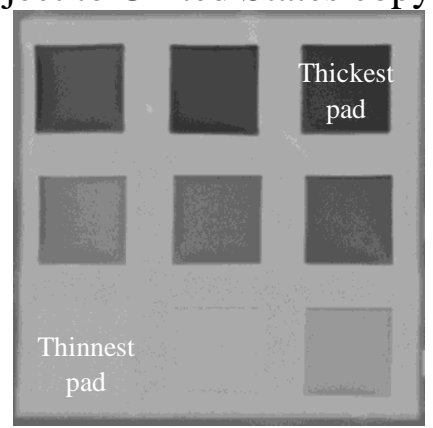

(c)

Figure 1. Annular dark-field images of ultrathin platinum pads at (a) $\beta=363 \mathrm{mrad}$, (b) $\beta=113 \mathrm{mrad}$, and (c) $\beta=42 \mathrm{mrad}$. Pads are $2 \mu \mathrm{m}$ square, and thickness increases from left to right and bottom to top.
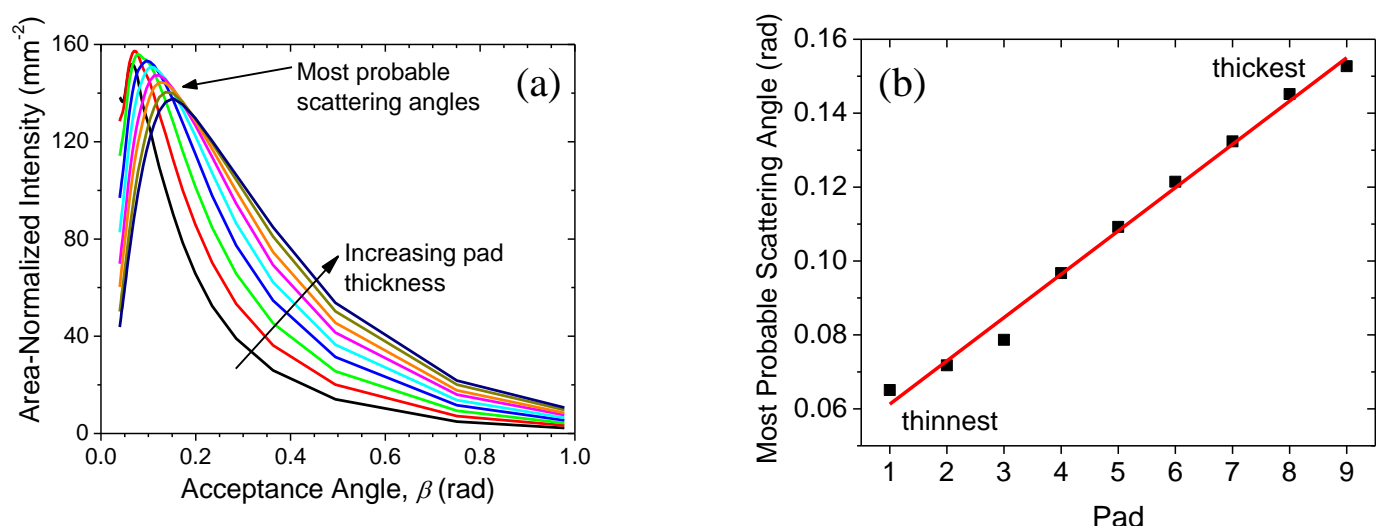

Figure 2. (a) Variation of the image intensity as a function of STEM detector acceptance angle, $\beta$, for the platinum sample. (b) The most probable scattering angle as a function of pad thickness. The red line is a guide for the eye. 